\title{
LA IMPLANTACIÓN DEL CUADRO DE MANDO INTEGRAL EN EL SECTOR AGROALIMENTARIO: EL CASO DEL GRUPO ALIMENTARIO GUISSONA*
}

\author{
ORIOL AMAT SALAS ${ }^{* *}$, LUCIA-CLARA BANCHIERI ${ }^{* * * *} \&$ FERNANDO CAMPA-PLANAS**** \\ UNIVERSITAT POMPEU FABRA (ESPAÑA) - UNIVERSIDAD NACIONAL DEL SUR (ARGENTINA) - UNIVERSITAT \\ ROVIRA I VIRGILI (ESPAÑA)
}

Recibido/ Received/ Recebido: 30/11/14 - Aceptado/ Accepted / Aprovado: 26/10/15

\begin{abstract}
Resumen
El Grupo Alimentario Guissona (ubicado en la provincia de Lleida en España), nació con una visión estratégica que le ha llevado a posicionarse con notable éxito en el mercado. Así mismo ha implementado diversas herramientas de gestión que le han permitido desarrollarse, adaptarse y crecer durante más de 50 años. El presente artículo plantea la implantación de un Cuadro de Mando Integral (CMI) para poder monitorear la referida estrategia. Se sugiere implementar esta herramienta concreta, ya que es la quinta herramienta de gestión más utilizada en el mundo según el estudio bianual que realiza Bain \& Company (Rigby \& Bilodeau, 2013). La propuesta de CMI contenida en el presente estudio incluye 27 indicadores, clasificados en las 4 perspectivas propuestas en el CMI. Palabras clave: Indicadores de gestión; Cuadro de mando integral; Toma de decisiones; Cooperativas agroalimentarias; Sector agroalimentario.
\end{abstract}

\section{THE IMPLEMENTATION OF THE BALANCED SCORECARD IN THE AGRI-FOOD SECTOR: THE CASE OF FOOD GROUP GUISSONA}

\begin{abstract}
The Guissona Food Group (located in the province of Lleida in Spain), was born with a strategic vision that has led it to position itself with remarkable success in the market. It has also implemented several management tools that have enabled the company to develop, adapt and grow for more than 50 years. This article discusses the implementation of a Balanced Scorecard (BSC) to monitor the aforementioned strategy. It is suggested to implement this particular tool, since it is the fifth most used management tool in the world according to the biannual study conducted by Bain \& Company (Rigby \& Bilodeau, 2013). BSC's proposal contained in this study includes 27 indicators, ranked the 4 perspectives proposed in the BSC.

Keywords: Management indicators; Balanced Scorecard; Decision making; Agribusiness cooperatives; Food industry.

\footnotetext{
Artículo de investigación científica interinstitucional.

** Profesor Universitat Pompeu Fabra, Barcelona. Dirección postal: Plaça de la Mercè, 10-12. 08002 Barcelona (España) Teléfono: (+34) 9354220 0. Correo electrónico: oriol.amat@upf.edu.

*** Profesora Universidad Nacional del Sur, Bahía Blanca (Argentina).

***** Profesor Universitat Rovira i Virgili, Reus (España).
} 


\title{
A IMPLANTAÇÃO DO QUADRO DE COMANDO INTEGRAL NO SETOR AGROALIMENTAR: O CASO DO GRUPO ALIMENTAR GUISSONA
}

\begin{abstract}
Resumo
O Grupo Alimentar Guissona (localizado na província de Lleida em Espanha) nasceu com uma visão estratégica que o levou a se posicionar com notável sucesso no mercado. Desta maneira tem implementado diversas ferramentas de gerenciamento que lhe permitiram se desenvolver, se adaptar e crescer durante mais de 50 anos. O presente artigo propõe a implantação de um Quadro de Comando Integral (CMI, por sua sigla em espanhol) para poder monitorar a referida estratégia. Sugere-se implementar esta ferramenta concreta, visto que é a quinta ferramenta de gerenciamento mais utilizada no mundo segundo o estudo bianual que realiza Bain \& Company (Rigby \& Bilodeau, 2013). A proposta de CMI contida no presente estudo inclui 27 indicadores, classificados em 4 perspectivas propostas.

Palavras chave: Indicadores de gerenciamento; Quadro de comando integral; Tomada de decisões; Cooperativas agroalimentares; Setor agroalimentar.
\end{abstract}

Amat, O., Banchieri, L-C. \& Campa-Planas, F. (2016). La implantación del cuadro de mando integral en el sector agroalimentario: el caso del Grupo Alimentario Guissona. En: Revista de la Facultad de Ciencias Económicas de la Universidad Militar Nueva Granada. rev.fac.cienc.econ, XXIV No1, DOI: $h$ ttp://dx.doi.org/10.18359/rfce.1619.

JEL: $M 41, M 49$.

\section{Introducción}

El Grupo Alimentario Guissona ubicado en el municipio del mismo nombre en la provincia de Lleida en Catalunya (España), fue creado en 1959 y es hoy en día un grupo de referencia en el sector agroalimentario español, teniendo integrados los procesos de actividad agrícola, ganadera, industrial y comercial, de forma que le permite llegar al consumidor final sin intermediarios.

Este Grupo ha implementado diversas estrategias y modelos de gestión para su crecimiento y supervivencia. Entre ellos, se encuentra la planificación estratégica, el CRM (Customer Relationship Management), encuestas de satisfacción del personal y el Benchmarking. Estas dos herramientas se incluyen entre las herramientas de gestión más utilizadas a nivel mundial (Rigby \& Bilodeau, 2013). La quinta herramienta de gestión más utilizada es el Balanced Scorecard (en castellano, y en adelante, Cuadro de Mando Integral - CMI).

Este artículo propone un CMI para la división de tiendas del Grupo Alimentario Guissona.
El documento está organizado en cuatro apartados. En primer lugar, se describe el Grupo Alimentario Guissona, su historia y sus factores diferenciales. A continuación, se desarrolla el modelo Cuadro de Mando Integral (CMI) y su utilidad. En tercer lugar, se desarrolla la propuesta de indicadores que contendría el CMI aplicable, organizados por cada una de las perspectivas propuestas por Kaplan y Norton (1992). Por último, se exponen las principales conclusiones derivadas del presente artículo.

\section{El Grupo Alimentario Guissona}

El Grupo Alimentario Guissona está integrado por la Cooperativa Agropecuaria de Guissona, la Caja Rural de Guissona, la Corporación Alimentaria Guissona, las fundaciones Agropecuaria y bonArea, Servicios Agropecuaria de Seguros y Servicios Médicos Guissona; siendo el grupo de capital catalán más importante en el sector de la alimentación. A nivel estatal, es el segundo del sector cárnico y del sector de piensos. La Corporación es la primera empresa de la provincia de Lleida (donde está ubicada geográficamente) y la primera empresa alimentaria 
española por volumen de facturación en España. Si incluimos las multinacionales, pasa a ser la quinta empresa alimentaria.

La Cooperativa tiene 12.040 socios, que son agricultores y ganaderos, y el Grupo tiene unos cuatro mil puestos de trabajo directos y mil quinientos más en las tiendas franquiciadas.

\subsection{Breve historia del Grupo}

La Cooperativa Avícola de Guissona (CAG) fue fundada en el año 1959 cuando un grupo de pioneros de la comarca de la Segarra (Lleida) liderados por Jaume Alsina, el actual presidente, iniciaron la actividad ganadera para complementar los ingresos fluctuantes de las actividades agrícolas. Crearon la cooperativa para proveerse de materias primas para sus granjas y, más adelante, para comercializar sus productos.

En el año 1962, los mismos socios crearon Caixa Guissona, caja rural para dar servicios financieros a los socios y a la Cooperativa. Caixa Guissona se caracteriza por una política prudente de riesgo que ha supuesto que sólo tenga en 2013 un 1,6\% de tasa de morosidad, cuando en los bancos y cajas españoles este ratio supera, actualmente, el $7 \%$. Su ratio de capital principal es del $26,1 \%$ superando ampliamente el $8 \%$ de mínimo requerido por las autoridades monetarias.

La primera fábrica de piensos de Guissona se construyó en 1962. Hoy, ya dispone de nueve fábricas en las provincias de Lleida, Tarragona, Barcelona y Zaragoza. La producción de alimentación animal es la única actividad industrial geográficamente dispersa (todas las demás se concentran en el municipio de Guissona), para acercar el suministro a las granjas.

En el año 1964 empiezan a comercializar huevos, cerdos y pollos; en 1965 se empiezan a prestar servicios técnicos para el ganado y abonos para el campo; en 1966 ponen en marcha una flota de vehículos propios y los contratos con los socios para la producción de pollos y de otros animales, en 1967 ya disponen de mataderos propios y en 1975 inician la actividad de preparados cárnicos.
Antes de llegar a la fórmula de tiendas propias, se ensayaron muchas fórmulas de comercialización, como la venta a domicilio, la venta cash a profesionales, etc. Las primeras tiendas bonArea (nombre comercial por el que son conocidas las tiendas de distribución del Grupo), se abrieron en el año 1994. Este era un reto importante, puesto que suponía competir con los clientes de la Cooperativa de entonces, las cadenas de supermercados. Al abrir las tiendas propias se perdieron los supermercados como clientes, pasando a vender directamente al cliente final.

De esta manera, Guissona completa el ciclo llegando a los consumidores sin intermediarios. Actualmente vende el $80 \%$ de la producción propia a partir de las 422 tiendas bonArea. El perfil de las tiendas es de una superficie de 180 metros cuadrados, aproximadamente, y situados en barrios o localidades de más de 10.000 habitantes. A partir de 2004 abren supermercados bonArea, con locales de hasta 500 metros cuadrados y, actualmente, son más de 130 establecimientos. Estos supermercados incorporan, entre otros, productos secos de alimentación y artículos de limpieza producidos por otros fabricantes. El Grupo ha hecho una clara apuesta por este formato grande. Incluso se estimula la transformación de tiendas normales a 'super bonArea'. Es una decisión estratégica que introduce plenamente al Grupo en el sector de la distribución y, por lo tanto, a competir directamente con las grandes cadenas de supermercados. Cada semana pasan por alguna de las tiendas bonArea 1,2 millones de clientes, el $85 \%$ de los cuales son catalanes.

Otra apuesta del grupo han sido las gasolineras. La primera se puso en marcha en el año 1991 y hoy ya tiene 40 gasolineras.

El grupo también hace varias actuaciones de tipo social como la esponsorización de actividades deportivas de Guissona; asistencia a gente mayor a través de la Fundación Agropecuaria de Guissona; y la construcción de viviendas asequibles; entre otras.

A lo largo de estos años, el Grupo ha sido distinguido con varios premios, cómo el de Mejor Empresa de Alimentación Española entregado por el Ministerio de Agricultura en 2006. O el premio FUNDE por 
la creación de puestos de trabajo concedida por la Asociación de Empresarias, Directivas y Profesionales en 2010.

\subsection{Factores diferenciales del Grupo}

Entre los principales factores diferenciales del Grupo se pueden destacar los siguientes:

- Líder: Todo proyecto empresarial de éxito se caracteriza por contar con un líder de primer orden y este es el caso de Jaume Alsina, que en el año 1959 ya era el primer secretario de la cooperativa y que en la actualidad es el presidente de la entidad. Sin él, el Grupo no existiría. Ya desde los inicios, y tras finalizar los estudios de Veterinaria, Jaume Alsina tenía la visión estratégica de que tenía que llegar a ser una empresa de grandes dimensiones y siempre ha utilizado la planificación estratégica como herramienta de gestión. También es un líder innovador y prudente. Una de las fuentes de innovación ha sido sus viajes constantes a otros países para aprender, sobretodo, en empresas norteamericanas y europeas.

- Corresponsabilidad: Cada sección del Grupo es un centro de beneficios que compra y vende a precios de mercado, dentro o fuera del Grupo. Cada camión es una sociedad particular entre el camionero y la Cooperativa, que comparten los resultados. Lo mismo pasa con las tiendas, las gasolineras, etc. Los incentivos económicos y la dirección por objetivos ha sido uno de los elementos motivadores.

Adicionalmente, tiene establecidos "círculos de calidad", reuniones donde se encuentran los trabajadores de un mismo departamento, sección o tipología de trabajo similar, para intentar aportar propuestas de mejora en todos los sentidos (calidad, seguridad laboral, productividad, costes, mejora procesos, etc.). Posteriormente, estas propuestas son valoradas por los directivos y se ejecutan las que consideran aplicables (para fomentar el éxito se premia al grupo de trabajadores que ha sugerido la propuesta mas bien valorada). También existen "Equipos de
Progreso" donde responsables y/o trabajadores de proyectos y/o actividades comunes se encuentran periódicamente para desarrollar proyectos comunes, encontrar soluciones, establecer iniciativas, ejecución de acciones, etc. Se dispone también de buzones de sugerencias en los diferentes centros de trabajo.

- Un proyecto empresarial que implica a más de 50.000 personas: Se trata de los socios agropecuarios, los clientes agropecuarios, los trabajadores, los accionistas y los franquiciados. Guissona es la primera población española que tiene más inmigrantes que autóctonos. Los inmigrantes proceden de más de 48 países diferentes, sobre todo de Ucrania y Rumanía, y aun así no hay conflictos raciales. Uno de los motivos es que hay oferta de trabajo para todos gracias al Grupo Guissona puesto que la tasa de paro de la comarca es del $2 \%$, menos de una décima parte del $21 \%$ de paro que hay en España.

- Modelo de integración único en el mundo: El factor diferencial más relevante del grupo es el tener un proceso totalmente integrado, horizontal y verticalmente, del campo a la mesa sin intermediarios. Todo empieza en los campos de cereales y en la producción animal, y se completa con el sacrificio, elaboración y comercialización de toda la gama cárnica (pollos, cerdos, ternera, etc.).

- Concentración en el mercado nacional: Una de las claves de muchas empresas exitosas está en saber decir "no" a las diversificaciones que pueden comportar el entrar en negocios o mercados geográficos desconocidos. Por este motivo, el grupo no tiene previsto iniciar actividades fuera de España.

Productividad y calidad: La gestión directa del ciclo productivo completo y la eficiencia permite diferenciarse del resto de competidores. De una parte, la realización y el control de todo el ciclo productivo aseguran la calidad y seguridad alimentarias y que el producto llegue lo más rápido posible al consumidor. Por la otra, la pasión por la productividad explica sus costes reducidos. 
- Economías de escala: Para reducir costes, se sigue una estrategia de volumen. Cómo ejemplo se puede indicar el centro alimentario de La Closa (200.000 metros cuadrados), iniciado en 1971 y actualmente uno de los más grandes del mundo. Se sacrifican diariamente 150.000 animales que se transforman en más de un millón de bandejas y paquetes de producto de libre servicio que se comercializan a través de las tiendas propias.

- Cogeneración: Otra técnica para reducir costes de fabricación es la cogeneración de electricidad, vapor y agua caliente. La primera planta de cogeneración se puso en marcha el año 1993.

- Logística: El grupo dispone de red propia de vehículos, con 175 vehículos, para garantizar que las tiendas reposten los productos cada día con el fin de garantizar la máxima calidad. En Guissona se dispone de un almacén automatizado para 25.000 palets.

- Comité de reducción de costes: Desde hace muchos años este Comité tiene el objetivo de reducir los costes cada año entre un $5 \%$ y un $10 \%$. Esto hace que los precios del Grupo son a menudo un $30 \%$ inferiores a los de la competencia directa.

El Comité de reducción de costes actúa teniendo en cuenta la estrategia de futuro. De esta manera, se rechazan aquellos ahorros que pueden comprometer la calidad o la imagen de la empresa y, por lo tanto, comprometer las ganancias futuras a causa de unos ajustes inadecuados.

- Precios de mercado para los socios: A diferencia de muchas cooperativas que trabajan sin margen para poder ofrecer al socio proveedor precios por encima del mercado, la Cooperativa ha apostado por precios de mercado que permitan generar excedentes que financien las inversiones y el crecimiento.

La competitividad de una empresa depende de muchos factores pero uno de ellos es la capacidad de negociación con los proveedores a fin de obtener unas condiciones de precio adecuadas para sus compras. En el caso de las cooperativas coincide propietario (socio) y proveedor.

El proveedor, desde su posición de socio, tiene tendencia a reclamar una mejora en el precio dejando a la cooperativa en una situación más débil que su entorno o, incluso, sin recursos para invertir, comprometiendo así el futuro.

En el caso de Guissona, la identificación del precio pagado a los socios con el precio de mercado comporta, en primer lugar, establecer un criterio objetivo para la fijación de precios, criterio más fácil de gestionar.

En segundo lugar, sitúa a la cooperativa en al mercado dónde debe competir forzosamente y en igualdad de condiciones en un aspecto clave como son los costes de abastecimientos.

En tercer lugar, facilita la obtención de recursos para la autofinanciación, de tal manera que el no percibir a corto plazo unos ingresos más elevados por parte de los socios acaba aportando ganancias tangibles en el futuro de la empresa cooperativa y de sus asociados.

- Fondos reguladores: También se pueden destacar los mecanismos de seguridad que se han establecido para hacer frente a la volatilidad de los precios agrarios puesto que los contratos de producción de ganado disponen de unos fondos reguladores con el $50 \%$ de los excedentes que moderan el déficit de los años malos.

Considerando la importancia estratégica de los alimentos, se han impulsado políticas para moderar esta volatilidad: ayudas, regulación de mercados, cuotas a la producción, etc. Todo con el objetivo de garantizar el abastecimiento alimentario, la estabilidad y la moderación de los precios, además, lógicamente, de permitir obtener unas rentas suficientes a los agricultores.

Precios de venta bajos al trabajar sin intermediarios: Como ya se ha indicado, los precios de venta de sus productos son bastante más bajos 
(alrededor de un 30\%) que los de la competencia como consecuencia del proceso integrado que elimina intermediarios.

El grupo también vende en internet mediante una tienda virtual que vende más de 8.000 productos diferentes, de los cuales 300 son productos cárnicos de elaboración propia y 1.400 son otros productos alimentarios.

- Fórmula de franquicia para las tiendas: A través de la franquicia, y también de las tiendas propias, se ha podido llegar a las 422 tiendas, de las cuales el 3\% son propias del grupo, en un plazo muy corto de tiempo. Estas tiendas están situadas en Cataluña, Aragón, Castellón, Madrid, Guadalajara y Andorra. Una de las características de las tiendas es que funcionan en régimen de autoservicio lo cual facilita una compra rápida y ágil.

- Boca-oreja: El Grupo ha invertido muy poco en publicidad, ya que la discreción ha sido uno de los factores característicos. En cambio, se ha priorizado el turismo industrial a fin de que los visitantes de la fábrica, los socios y los clientes sean la mejor publicidad.

- Política financiera conservadora: Los primeros años fueron muy complicados y les dieron una buena lección de management ya que en palabras de Jaume Alsina: "Teníamos que basar nuestro futuro en la eficacia y en la propia solvencia, evitar riesgos innecesarios e invertir con recursos propios". Esta es otra característica habitual de las empresas con éxito, ya que se trata de un grupo de empresas muy capitalizado. Esto explica que prácticamente no tenga deudas financieras. La mayor parte de los excedentes se han destinado siempre a autofinanciación. Así, no ha hecho falta endeudarse para hacer inversiones y crecer. La política de dividendos ha sido, en términos generales, distribuir a los accionistas alrededor de un $20 \%$ de los beneficios. En 2013 tuvo unos ingresos de 1.467 millones de euros y unos beneficios de 37,3 millones de euros.

- Sistemas de información: La empresa siempre ha sido pionera en sistemas de información. En este sentido, en 1969 ya adquirió un ordenador IBM, lo cual era un hecho insólito durante esos años, ya que sólo los bancos y las aseguradoras disponían de estos medios. Así mismo tienen incorporado un sistema de CRM, para poder conocer la tipología de cliente y actuar en consecuencia al mismo, segmentando las correspondientes estrategias de marqueting.

En conjunto, se trata de una empresa que ha contribuido a generar valor para sus socios, trabajadores y accionistas, y que ha contribuido de una manera decisiva a elevar el nivel de renta de la agricultura catalana. Para lograrlo, la clave ha sido la integración de todo el proceso desde la producción hasta la comercialización y la pasión constante por la calidad y la productividad para ofrecer productos con una relación calidad-precio difícil de igualar.

\section{El Cuadro de Mando Integral (CMI)}

\subsection{El modelo: origen y evolución}

El Cuadro de Mando Integral nace en la década de los 90 del siglo pasado cuando Kaplan \& Norton (1992, 1993) comienzan a trabajar en un proyecto en común cuyo objetivo era explorar nuevas formas para medir el desempeño organizacional. Los autores estaban convencidos de que las ventajas competitivas de las organizaciones empezaban a asentarse cada vez más en las inversiones basadas en el conocimiento (en los empleados y en las tecnologías de información). Sin embargo, para medir su desempeño las organizaciones seguían recurriendo sólo al sistema de contabilidad financiera, aun cuando éste no proporcionaba base alguna para medir, analizar y gestionar el valor agregado que se lograba aumentando las capacidades de los llamados activos intangibles.

El Cuadro de Mando Integral agregó a la perspectiva financiera, que ya estaba siendo evaluada por las organizaciones, la perspectiva de los clientes, de los procesos internos y la perspectiva de aprendizaje y crecimiento, representando esta última, los activos intangibles de las organizaciones que son los que impulsan las ventajas competitivas (las personas, los sistemas y la cultura de la empresa). 
La herramienta fue incorporada rápidamente por diversas empresas, que demandaban aplicar el sistema para solucionar problemas importantes: cómo implementar nuevas estrategias, cómo lograr que los empleados comprendieran estas nuevas estrategias y las pudieran aplicar en su trabajo diario.

Así, el CMI evolucionó de un sistema de indicadores a un sistema de gestión centralizada. Kaplan y Norton (1992) lo definen como un modelo que "traduce la estrategia y la misión de una organización en un amplio conjunto de medidas de la actuación, que proporcionan la estructura necesaria para un sistema de gestión y medición estratégica". En esta dirección Lawrie \& Cobbold (2004) sostienen que el CMI ha evolucionado para convertirse en un modelo de gestión a largo plazo que elimina la distancia entre la formulación y la aplicación de la estrategia.

Como se mencionó anteriormente el modelo está compuesto por cuatro perspectivas, financiera, clientes, procesos internos y aprendizaje y crecimiento. Estas perspectivas están presentes en la mayoría de las organizaciones actuales independientemente del sector en el que operan. Kaplan \& Norton (1996) explican que en los casos en que la organización lo requiriera, podrían incorporarse perspectivas adicionales o prescindir de alguna de las establecidas en el modelo teórico.

Dicho CMI está evolucionando en los últimos años, de forma que diversos autores promueven la idea de incorporar una perspectiva que incorpore a sectores externos a la organización que el modelo inicial no contempla, como por ejemplo el sector público, la sociedad o el medioambiente (Epstein \& Manzoni, 1998; Nørreklit, 2000; Brignall, 2002; Figge et al, 2002; Van Der Woerd \& Van Der Brink, 2004).

Otro de los elementos claves del modelo, junto con las perspectivas, son las relaciones de causa-efecto. Estas relaciones vinculan a los objetivos de tal manera que describen la estrategia de la organización. Estas relaciones deben ser explícitas para que puedan ser gestionadas y confirmadas.

El CMI ha sido extensamente estudiado y ha recibido críticas: al modelo teórico en forma global, a las perspectivas, a los indicadores y a las relaciones de cau- sa-efecto. Sin embargo, es un modelo ampliamente implementado en el mundo (Banchieri et al, 2011).

En relación a la implantación del CMI en Catalunya (donde actúa principalmente el Grupo Alimentario, un estudio de Banchieri \& Campa-Planas (2010), concluía que el CMI es una herramienta conocida, ya que más del $57 \%$ de las empresas afirmaba conocerlo, pero sin embargo sólo el 15,7\% lo usaba.

\subsection{Utilidad del CMI}

Kaplan y Norton como creadores del CMI, resaltan, entre otras, dos utilidades del mismo (Kaplan \& Norton, 1996):

- El modelo provee información valiosa y ofrece una visión global del desempeño de la organización a los directivos.

- La aportación del modelo para la gestión integral de la estrategia, ya que permite: esclarecer y descifrar la visión y la estrategia; trasmitir y relacionar los objetivos e indicadores estratégicos; planificar, fijar los objetivos y encauzar las iniciativas relacionadas con la estrategia; $y$, acrecentar el feedback y la formación estratégica.

La tabla 1 menciona los autores que han destacado diversas aportaciones adicionales sobre el CMI.

Tabla 1. Aportaciones del CMI

\begin{tabular}{|l|c|}
\hline \multicolumn{1}{|c|}{ Utilidad } & \multicolumn{1}{|c|}{ Autor } \\
\hline $\begin{array}{l}\text { Provee información importante de forma concisa a la } \\
\text { Dirección. }\end{array}$ & \\
$\begin{array}{l}\text { Favorece el aprendizaje organizativo. } \\
\text { Es un sistema de control íntegro y facilita el diagnósti- } \\
\text { co ya que incorpora indicadores a largo plazo }\end{array}$ & $\begin{array}{c}\text { Mooraj et al. } \\
(1999) .\end{array}$ \\
\hline Permite gestionar la estrategia corporativa & $\begin{array}{c}\text { Leung et al. } \\
(2006) ; \text { Aparisi } \\
(2008) ; \text { De Geu- } \\
\text { ser et al. (2009). }\end{array}$ \\
\hline Herramienta de control & $\begin{array}{c}\text { Porporato \& Par- } \\
\text { kinson (2008). }\end{array}$ \\
\hline Permite vincular los objetivos con las remuneraciones & Quinlivan (2000). \\
\hline
\end{tabular}

Fuente: Elaboración propia. 
Amat et al., (2013) resumen la utilidad del CMI en que: brinda información relevante sobre las principales variables financieras y no financieras, comunica la estrategia a toda la organización permitiendo alinear los objetivos personales a los organizacionales, posibilita la formación, facilita la mejora continua y promueve la revisión de la estrategia empresarial $^{1}$.

\section{Misión y estrategia del Grupo Ali- mentario Guissona}

Con base en la misión de la Compañía, podemos determinar la estrategia de la empresa y, consecuentemente, el CMI a proponer, compuesto de una serie de indicadores que permitan monotorizar el éxito en la obtención de los objetivos propuestos. Para la determinación de dicho CMI, y consiguientes indicadores, debe definirse, entre otros aspectos, la estrategia en base a la misión de la compañía, de acuerdo a los siguientes pasos:

- Establecimiento de la misión: Según el Grupo, su misión es "mejorar el rendimiento de las explotaciones ganaderas y atender las necesidades de los clientes que confían en nuestra eficacia y profesionalidad, ofreciéndoles productos seguros, de la mejor calidad y al mejor precio. En el Grupo trabajamos para ofrecer a nuestros clientes el fruto de todo este proceso, productos con garantía de origen".

- Análisis interno y externo: En relación al análisis externo podemos indicar que derivada de la compleja situación en la que se encuentra inmersa la economía española, ha habido una disminución en la demanda y consecuente reducción de venta de productos alimenticios, hecho que ha supuesto un incremento de la competencia con la necesidad de diferenciación por producto, distribución y/o precio. En relación a este último aspecto y, vinculando ya con el análisis interno, destacar que los productos del grupo siguen una

Véase también Grau \& Sole (2013). estrategia donde la relación calidad-precio es su eje principal.

- Identificación de los factores clave para el éxito de la organización: El factor clave para la organización es mantener a los clientes satisfechos con sus compras, es decir con el producto que el grupo ha puesto a su disposición, ya que el objetivo seguido por la empresa es mantener una relación a largo plazo con los clientes, a través de la fidelización con sus productos, clientes y hábitos de compra.

- Relación causa-efecto entre los factores clave: En la figura 1, se muestra el mapa estratégico desarrollado por los autores para la identificación de las variables clave de la Organización, y su impacto en el logro de los objetivos estratégicos planteados.

- Establecimiento de los objetivos estratégicos: La organización tiene establecidos una serie de objetivos estratégicos, que se desarrollan en el apartado 5 de este trabajo, vinculados ya con los indicadores de seguimiento de los mismos.

\section{Propuesta de indicadores de gestión y CMI para el Grupo Alimentario Guissona}

En base a las características del Grupo Alimentario Guissona, su estrategia y considerando las ventajas e inconvenientes que se han expuesto respecto al modelo de CMI de Kaplan \& Norton, a continuación se describe una propuesta de indicadores que integrarían el CMI, para la unidad de negocio de venta minorista., el cual incluye la venta por internet, las tiendas bonArea y los super bonArea.

\subsection{Perspectiva económica-financiera}

Como se explicó anteriormente, los indicadores económicos financieros son analizados y monitoreados por todos los directivos. Para Kaplan y Norton (1996) estos indicadores no sólo muestran los resultados de la gestión financiera de la organización que se espera según la estrategia sino que también sirven forma de medir los resultados de las demás perspectivas del CMI. 
Figura 1. Propuesta de Mapa estratégico

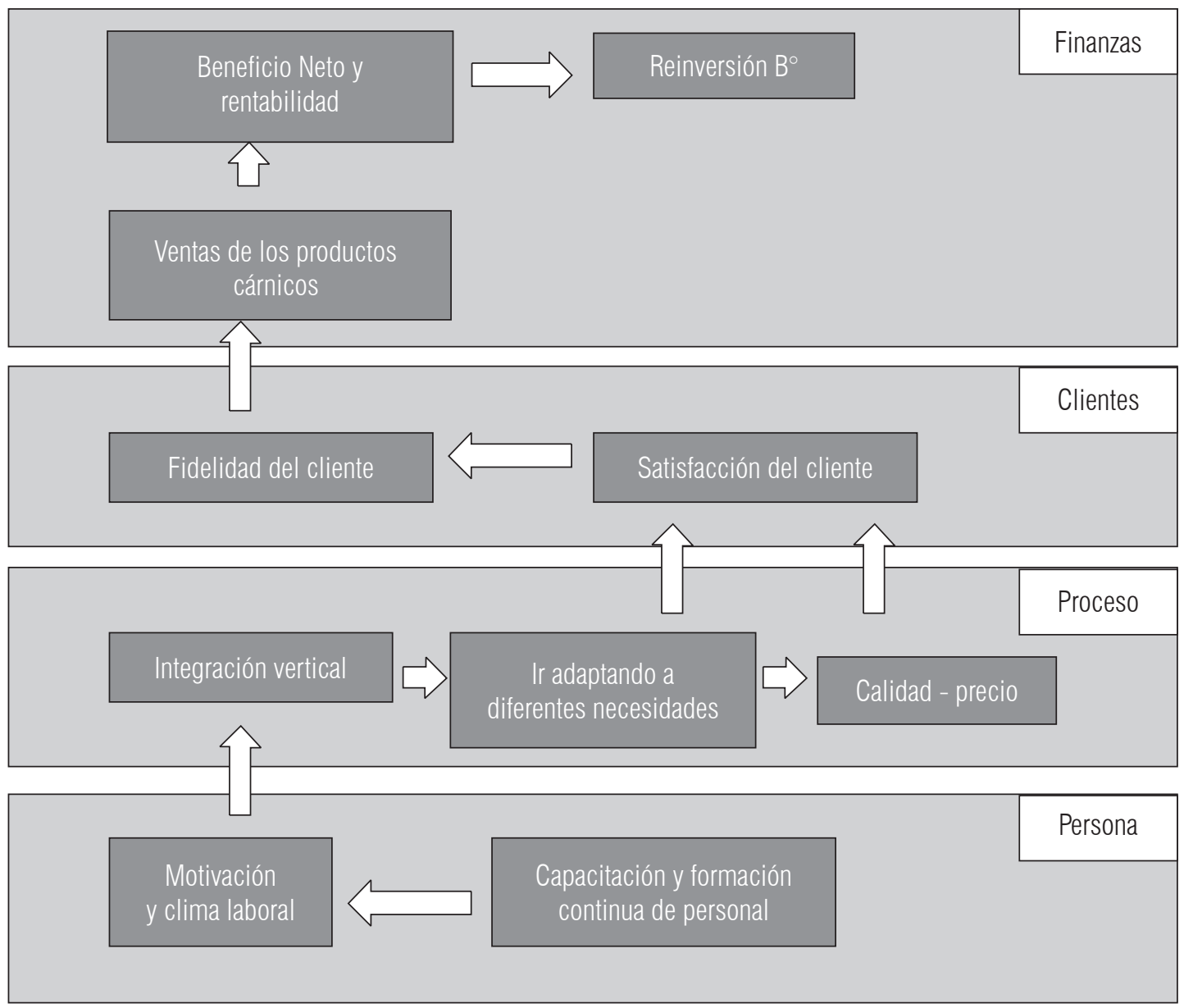

Fuente: Elaboración propia.

Los autores identifican tres temas estratégicos para poder monitorear los aspectos relevantes de la perspectiva financiera:

- Crecimiento y diversificación de los ingresos: ello se alcanzará ofreciendo nuevos servicios y/o productos/procesos, atrayendo a nuevos clientes, llegando a nuevos mercados, cambiando la mezcla de productos/procesos y/o servicios para ofrecer una propuesta de mayor valor añadido y cambiando el precio de dichos productos o servicios.

- Reducción de costos/mejora en la productividad: es la reducción de costos directos e indirectos y el compartir recursos comunes con otras unidades de negocio. Este aspecto permite controlar los costos para que guarden la relación establecida con los ingresos, con la producción $\mathrm{y}$ con los procesos.

- Utilización de los activos/estrategia de la inversión: este objetivo se centra en utilizar los activos (físicos y financieros) de una manera más eficiente, para poder obtener una mayor rentabilidad de los mismos, a través de la correspondiente mejora en producto y proceso.

Los indicadores propuestos en esta perspectiva a nivel global del negocio de distribución en tiendas se acompañan en la tabla 2. 
Tabla 2. Indicadores de la perspectiva Económica-financiera

\begin{tabular}{|l|l|}
\hline \multicolumn{1}{|c|}{ Indicador } & \\
\hline Ventas & Indica el importe de facturación por canal de venta. \\
\hline Nuevas tiendas & $\begin{array}{l}\text { Número de tiendas propias o franquicias abiertas en el período, que permita analizar la evolución de la Rentabilidad } \\
\text { Económica }\end{array}$ \\
\hline Markup o margen & $\begin{array}{l}\text { Indica el margen de beneficio bruto de la organización por la venta realizada. Es la diferencia entre la facturación y el coste de } \\
\text { la mercadería vendida. }\end{array}$ \\
\hline Gasto de personal & Muestra el porcentaje de la facturación que representa el gasto de personal. \\
\hline Gastos de funcionamiento & Describe el porcentaje de la facturación que representan los gastos de funcionamiento. \\
\hline Rentabilidad sobre el Patrimonio Neto & Indica el rendimiento obtenido de los fondos propios \\
\hline Rentabilidad económica & Indica el rendimiento de los activos de la organización \\
\hline Liquidez o Prueba ácida & $\begin{array}{l}\text { Muestra la capacidad de la empresa para cancelar sus deudas con sus activos corrientes sin considerar la venta de la } \\
\text { mercadería. }\end{array}$ \\
\hline
\end{tabular}

Fuente: Elaboración propia.

\subsection{Perspectiva de clientes}

Esta perspectiva identifica los segmentos de clientes y el mercado en el que quiere actuar la organización, de forma que le permitan producir mejores rendimientos financieros futuros. Aspira a la satisfacción y retención de los clientes. Incluye los indicadores de nuevos clientes, rentabilidad por cliente, cuota de mercado y factores por los cuales el cliente será fiel al servicio o al producto (calidad, tiempo, precio, entre otros).

El modelo contempla que para retener o captar clientes en un mercado cada vez más competitivo debe ofrecerse una propuesta de valor destinada al mercado objetivo que combine los atributos del servicio o producto, con la relación entre la organización y los clientes, y la imagen de la entidad (tabla 3).

Tabla 3. Indicadores de la perspectiva de clientes

\begin{tabular}{|l|l|}
\hline \multicolumn{1}{|c|}{ Indicador } & \multicolumn{1}{c|}{ Descripción } \\
\hline Cuota de Mercado & $\begin{array}{l}\text { Indica la proporción del total de ventas de las poblaciones en que se encuentra la organización que le corresponde, en } \\
\text { relación a otros puntos de venta. }\end{array}$ \\
\hline Posicionamiento de precios & $\begin{array}{l}\text { Muestra la situación de los precios de venta de la organización en relación a los de su competencia. Para ello deberían } \\
\text { determinarse X productos representativos. }\end{array}$ \\
\hline Calidad de los productos & Indica la proporción de productos retirados de la venta o no exhibidos por problemas de calidad o de caducidad \\
\hline Satisfacción del cliente & $\begin{array}{l}\text { Describe la satisfacción de los clientes en una escala prefijada por la Organización. El valor del indicador se obtiene de una } \\
\text { encuesta que se realiza entre los consumidores in-situ. }\end{array}$ \\
\hline Ticket promedio & Muestra el promedio del monto gastado en una compra. \\
\hline Promedio de unidades & Indica la cantidad promedio de unidades vendidas por cada ticket de compra \\
\hline
\end{tabular}

Fuente: Elaboración propia.

\subsection{Perspectiva de Procesos Internos}

Se identifican a través de esta perspectiva los procesos internos críticos en los cuales la organización debe destacarse, mediante aquellos procesos que crean valor para los clientes. Los indicadores en esta perspectiva, se centran en aquellos procesos que impactan directamente en la satisfacción de las necesidades del consumidor y en el cumplimiento de los objetivos financieros de la empresa (tabla 4). 
Tabla 4. Indicadores de la perspectiva de procesos internos

\begin{tabular}{|l|l|}
\hline \multicolumn{1}{|c|}{ Indicador } & \multicolumn{1}{|c|}{ Descripción } \\
\hline Innovación & $\begin{array}{l}\text { Cantidad relativa de nuevos productos o } \\
\text { servicios ofrecidos. }\end{array}$ \\
\hline Abastecimiento & $\begin{array}{l}\text { Número de roturas de stocks, por faltas de } \\
\text { producto en tienda }\end{array}$ \\
\hline Marca propia & $\begin{array}{l}\text { Proporción de los productos vendidos que } \\
\text { son de producción propia y con visibilidad } \\
\text { (marca) propia }\end{array}$ \\
\hline Reclamaciones solucionadas & $\begin{array}{l}\text { Es la razón entre la cantidad de reclamos } \\
\text { recibidos y resueltos sobre la cantidad de } \\
\text { reclamos recibidos en un período deter- } \\
\text { minado }\end{array}$ \\
\hline Insumos propios & $\begin{array}{l}\text { Proporción de los insumos que son provis- } \\
\text { tos por empresas del grupo (energía, com- } \\
\text { bustibles, materias primas, etc.) }\end{array}$ \\
\hline Rotación de existencias & $\begin{array}{l}\text { Cantidad de operaciones realizadas por } \\
\text { cada canal de venta. }\end{array}$ \\
\hline Operaciones & $\begin{array}{l}\text { Indica los días promedio de existencias de } \\
\text { que se dispone en las tiendas. }\end{array}$ \\
\hline $\begin{array}{l}\text { Indica la proporción de productos retirados } \\
\text { de la venta 0 no exhibidos por problemas } \\
\text { de calidad o de caducidad }\end{array}$ \\
\hline Cactos
\end{tabular}

Fuente: Elaboración propia.

\subsection{Perspectiva de aprendizaje y crecimiento}

Identifica aquellos procesos vinculados a las personas, sistemas y procedimientos que la organización no sólo no puede descuidar sino que debe fomentar para crecer, mejorar y ser rentable en el largo plazo.

Aprendizaje y crecimiento proporciona la "infraestructura" para que el resto de las perspectivas puedan alcanzar sus objetivos. Esta perspectiva trata de identificar los activos intangibles de la organización y cómo se deberían comportar los mismos para lograrla estrategia (tabla 5).
Tabla 5. Indicadores de la perspectiva de aprendizaje y crecimiento

\begin{tabular}{|l|l|}
\hline \multicolumn{1}{|c|}{ Indicador } & \multicolumn{1}{c|}{ Descripción } \\
\hline Rotación de empleados & Antigüedad promedio de la plantilla \\
\hline Productividad & Indica la facturación por empleado \\
\hline $\begin{array}{l}\text { Salario promedio de los } \\
\text { empleados }\end{array}$ & $\begin{array}{l}\text { Cálculo del coste promedio por emplea- } \\
\text { do, comparable con el salario medio en } \\
\text { el sector }\end{array}$ \\
\hline Rotación de empleados & $\begin{array}{l}\text { Número de empleados que han mar- } \\
\text { chado de la empresa en un ejercicio, en } \\
\text { relación al total plantilla }\end{array}$ \\
\hline Fidelidad de empleados & Antigüedad media de los empleados \\
\hline Satisfacción del personal & $\begin{array}{l}\text { Muestra en una escala determinada el } \\
\text { grado de satisfacción del personal obte- } \\
\text { nido de una encuesta periódica de clima } \\
\text { laboral }\end{array}$ \\
\hline $\begin{array}{l}\text { Porcentaje de Sugerencias } \\
\text { implementadas }\end{array}$ & $\begin{array}{l}\text { Cantidad de sugerencias realizadas por } \\
\text { el personal que la organización decidió } \\
\text { implementar sobre la cantidad total de } \\
\text { sugerencias realizadas }\end{array}$ \\
\hline $\begin{array}{l}\text { Disponibilidad de sistemas de } \\
\text { información }\end{array}$ & $\begin{array}{l}\text { Tiempo de no disponibilidad de los sis- } \\
\text { temas de información vinculados a ne- } \\
\text { gocio, en el período analizado }\end{array}$ \\
\hline $\begin{array}{l}\text { Inversión en sistemas de } \\
\text { información }\end{array}$ & $\begin{array}{l}\text { Proporción de las ventas que se invierte } \\
\text { en los sistemas de la organización }\end{array}$ \\
\hline
\end{tabular}

Fuente: Elaboración propia.

Se propone utilizar el indicador de sugerencias realizadas e implementadas ya que es una forma de medir la motivación y el compromiso de los empleados con la organización y la mejora de sus procesos.

\section{Conclusiones}

El Grupo Alimentario Guissona es una referencia en Catalunya, que en sus 55 años de historia ha conseguido posicionarse como un Grupo de referencia a nivel de solvencia económica y como un caso de éxito por la aplicación de técnicas de gestión e integración de los trabajadores en su modelo de empresa, el cual se ha venido renovando constante, $y$ consecuentemente, durante su historia.

En su análisis se observa como el Grupo ha ido renovando su estrategia, pasando de ser productor y distribuidor al canal de venta, a ser distribuidor al 
cliente final, convirtiéndose en competidor de los que, hasta aquel momento, habían sido sus clientes. Así mismo, ha introducido nuevos servicios y productos con la idea de complementar y diversificar su oferta: Por lo tanto, se observa una empresa que analiza y adapta su estrategia al entorno competitivo, facilitando la participación de sus empleados en la gestión de la misma.

En base a dicha estrategia, y siguiendo el modelo de análisis de Kaplan y Norton y su conocido Cuadro de Mando Integral, se propone un modelo de indicadores para el negocio de tiendas, de ventas al cliente final; de forma que se permita medir la validez y éxito de dicha estrategia. Dicho modelo de indicadores, en cuanto está vinculado a la estrategia no es transferible a otra Organización similar (distribución alimentaria), al mismo tiempo que debería ser replanteado por el Grupo en caso de cambio en su estrategia actual.

Resaltar que para la implantación y seguimiento de un modelo de CMI es imprescindible la involucración y liderazgo de la Dirección de una Compañía. Sin duda, el perfil de su Director, Jaume Alsina, es garantía de éxito en caso de llevar a cabo la referida implantación.

Finalmente, sería interesante como futura línea de investigación, en caso que el CMI fuera desarrollado por el Grupo, como así en principio está planteado, examinar los resultados de la aplicación del CMI, recopilando factores que hayan sido considerado clave para la esperada exitosa implantación de dicho CMI.

\section{Referencias}

Amat, O., Campa-Planas, F. \& Fernández, A. (2013) "Cuadro de Mando Integral" En: Amat, O. \& Campa-Planas, F. (Coord.). Manual del Controller. Asociación Catalana de Contabilidad y Dirección. Barcelona: Profit Editorial.

Aparisi Caudeli, J. A. (2008). La eficacia del Cuadro de Mando Integral en el control estratégico de las entidades públicas. Valencia: Sindicatura de Comptes.

Banchieri, L.C. \& Campa-Planas, F. (2010). "La utilización del Cuadro de Mando Integral en Cataluña". VI Congreso Iberoamericano de Administración Empresarial y Contabilidad y IV Congreso Iberoamericano de Contabilidad de Gestión. Valencia, 7-9 de Junio de 2010.
Banchieri, L.C., Campa-Planas, F. \& Sánchez-Rebull, M.V. (2011) "What has been said, and what remains to be said, about the Balanced Scorecard?". En Proceedings of Rijeka Faculty of Economics Journal of Economics and Business, 29 (1): 155-192.

Brignall, T.J. (2002) "The unbalanced scorecard: a social and environmental critique". En: Performance Measurement and Management (2002): research and action: papers from the Third: 85-92.

De Geuser, F., Mooraj, S. \& Oyon, D. (2009) "Does the Balanced Scorecard Add Value? Empirical Evidence on its Effect on Performance". En European Accounting Review, 18 (1): 93-122.

Epstein, M. \& Manzoni, J. (1998) "Implementing corporate strategy: From tableaux de Bord to Balanced Scorecard". En: European Management Journal, 16 (2): 190-203.

Figge, F., Hahn, T., Schaltegger, S. \& Wargner, M. (2002) "The sustainability balanced scorecard - linking sustainability management to business strategy". En Business Strategy and the Environment, (11): 269-284.

Grau, A. \& Sole, M. F. (2013) "La retribución variable" En: Amat, O. y Campa-Planas F. (Coord.). Manual del Controller. Asociación Catalana de Contabilidad y Dirección. Barcelona: Profit Editorial.

Kaplan, R. S. \& Norton, D. P. (1992) "The Balanced ScorecardMeasures That Drive Performance". En: Harvard Business Review, 70 (1): 71-79.

Kaplan, R. S. \& Norton, D. P. (1993) "Putting the Balanced Scorecard to Work". En Harvard Business Review, 71 (5): 134-147.

Kaplan, R. S. \& Norton, D.P. (1996). The Balanced Scorecard: Translating strategy into action. Boston Massachusetts: Harvard Business School Press.

Lawrie, G. \& Cobbold, I. (2004) "Third-generation balanced scorecard: evolution of an effective strategic control tool". En: International Journal of Productivity and Performance Management, 53 (7): 611-623.

Leung, L. C., Lam, K. C. \& Cao, D. (2006) "Implementing the balanced scorecard using the analytic hierarchy process \& the analytic network process". En: Journal of the Operational Research Society, 57 (6): 682-691.

Mooraj, S., Oyon, D. \& Hostettler, D. (1999) "The Balanced Scorecard: a Necessary Good or an Unnecessary Evil?" En: European Management Journal, 17 (5): 481.

Nørreklit, H. (2000) "The balance on the balanced scorecard-a critical analysis of some of its assumptions". En: Management Accounting Research, 11 (1): 65-88.

Porporato, M. \& Parkinson, J. (2008) "Una investigación empírica sobre los factores que afectan a la selección de medidas del Cuadro de Mando Integral". En: Revista Iberoamericana de Contabilidad de Gestión, 6 (12).

Quilivan, D. (2000) "Rescaling the Balanced Scorecard for Local Government”. En: Australian Journal of Public Administration, 59 (4): 36-41.

Rigby, D. \& Bilodeau, B. (2013) "Management Tools and Trends 2013". Disponible en: http://www.bain.com/Images/BAIN BRIEF_Management_Tools_\%26_Trends_2013.pdf [consultado 7 de Noviembre de 2014$]$.

Van Der Woerd, F. \& Van Den Brink, T. (2004) "Feasibility of a Responsive Business Scorecard--a pilot study". En: Journal of Business Ethics, 55 (2): 173-186. 